\title{
Mining severe drug-drug interaction adverse events using Semantic Web technologies: a case study
}

\author{
Guoqian Jiang ${ }^{*}$, Hongfang Liu, Harold R Solbrig and Christopher G Chute
}

\author{
* Correspondence: \\ jiang.guoqian@mayo.edu \\ Department of Health Sciences \\ Research, Mayo Clinic, Rochester, \\ MN, USA
}

\begin{abstract}
Background: Drug-drug interactions (DDIs) are a major contributing factor for unexpected adverse drug events (ADEs). However, few of knowledge resources cover the severity information of ADEs that is critical for prioritizing the medical need. The objective of the study is to develop and evaluate a Semantic Web-based approach for mining severe DDI-induced ADEs.

Methods: We utilized a normalized FDA Adverse Event Report System (AERS) dataset and performed a case study of three frequently prescribed cardiovascular drugs: Warfarin, Clopidogrel and Simvastatin. We extracted putative DDI-ADE pairs and their associated outcome codes. We developed a pipeline to filter the associations using ADE datasets from SIDER and PharmGKB. We also performed a signal enrichment using electronic medical records (EMR) data. We leveraged the Common Terminology Criteria for Adverse Event (CTCAE) grading system and classified the DDI-induced ADEs into the CTCAE in the Web Ontology Language (OWL).

Results: We identified 601 DDI-ADE pairs for the three drugs using the filtering pipeline, of which 61 pairs are in Grade 5, 56 pairs in Grade 4 and 484 pairs in Grade 3. Among 601 pairs, the signals of 59 DDI-ADE pairs were identified from the EMR data.

Conclusions: The approach developed could be generalized to detect the signals of putative severe ADEs induced by DDIs in other drug domains and would be useful for supporting translational and pharmacovigilance study of severe ADEs.
\end{abstract}

Keywords: Drug-drug Interaction, Adverse drug event, Data mining, Semantic web technology, Electronic medical records

\section{Biomed Central}

\section{Introduction}

Drug-drug interactions (DDIs) are a major contributing factor for unexpected adverse drug events (ADEs) [1]. A semantically coded knowledge base of DDI-induced ADEs with severity information is critical for clinical decision support systems and translational research applications. In particular, there is emerging interest in investigating genetic susceptibility of DDI-induced ADEs and developing genetic tests to identify all those at risk of ADEs prior to prescribing potentially dangerous medication [2,3], in which the severity information is essential for prioritizing the medical need to evaluate the potential impact of pharmacogenomics information in reducing ADEs [4]. However, few of knowledge resources cover severity information of ADEs.

(c) 2015 Jiang et al.; licensee BioMed Central. This is an Open Access article distributed under the terms of the Creative Commons Attribution License (http://creativecommons.org/licenses/by/4.0), which permits unrestricted use, distribution, and reproduction in any medium, provided the original work is properly credited. The Creative Commons Public Domain Dedication waiver (http:// creativecommons.org/publicdomain/zero/1.0/) applies to the data made available in this article, unless otherwise stated. 
While recognizing, explaining and ultimately predicting DDIs constitute a huge challenge for medicine and public health, informatics-based approaches are increasingly used in dealing with the challenge [5]. Semantic Web technologies provide a scalable framework for data standardization and data integration from heterogeneous resources. For instance, Samwald et al. [6] developed a Semantic Web-based knowledge base for query answering and decision support in clinical pharmacogenetics, in which three dataset components are integrated. In our previous and ongoing study, we developed a standardized knowledge base of ADEs known as ADEpedia (http://adepedia.org) leveraging Semantic Web technologies [7]. The ADEpedia is intended to integrate existing known ADE knowledge for drug safety surveillance from disparate resources such as Food and Drug Administration (FDA) Structured Product Labeling (SPL) [7], FDA Adverse Event Reporting System (AERS) [8], and the Unified Medical Language System (UMLS) [9].

The objective of the study is to develop and evaluate a Semantic Web-based approach for mining severe DDI-induced ADEs. We utilized a normalized FDA AERS dataset and performed a case study of three frequently prescribed cardiovascular drugs: Warfarin, Clopidogrel and Simvastatin. We extracted putative DDI-ADE pairs and their associated outcome codes. We developed a pipeline to filter the associations using ADE datasets from SIDER and PharmGKB. We also performed a signal enrichment using electronic medical records (EMR) data. We leveraged the Common Terminology Criteria for Adverse Event (CTCAE) grading system and classified the DDI-induced ADEs into the CTCAE in the Web Ontology Language (OWL).

\section{Background}

\section{FDA Adverse Event Reporting System (AERS)}

FDA AERS is a database that provides information on adverse event and medication error reports submitted to FDA [10]. By the definition of FDA, the "serious" means that one or more of the following outcomes were documented in the report: death (DE), hospitalization (HO), life threatening (LT), disability (DS), congenital anomaly (CA) and/ or other (OT) serious outcome. In our previous study, we produced a normalized AERS dataset known as AERS-DM [11]. The dataset contains 4,639,613 unique putative DrugADE pairs in which the drugs are represented by RxNorm [12] codes and the putative ADEs are represented by MedDRA [13] codes. The data set also contains the unique ID number (known as ISR) for each corresponding AERS report, which is a primary link field between the AERS data file. We used the ISR field to identify the outcome codes of each AERS report. Table 1 shows the outcome code definitions in AERS database.

Table 1 Outcome code definitions in AERS database

\begin{tabular}{ll}
\hline Outcome code & Definition \\
\hline DE & Death \\
LT & Life-Threatening \\
HO & Hospitalization - Initial or Prolonged \\
DS & Disability \\
CA & Congenital Anomaly \\
RI & Required Intervention to Prevent Permanent Impairment/Damage \\
OT & Other \\
\hline
\end{tabular}




\section{Common Terminology Criteria for Adverse Event (CTCAE)}

CTCAE is a widely accepted, standard grading scale for adverse events throughout the oncology research community [14]. The current released version is CTCAE 4.0. This version contains 764 AE terms and 26 "Other, specify" options for reporting text terms not listed in CTCAE. Each AE term is associated with a 5-point severity scale. The AE terms are grouped by MedDRA Primary SOC classes. In the CTCAE, "Grade" refers to the severity of the adverse event (AE). The CTCAE displays Grades 1 through 5 with unique clinical descriptions of severity for each $\mathrm{AE}$ based on a general guideline. Table 2 shows the grade definitions in the CTCAE grading system.

\section{ADE datasets}

SIDER (SIDe Effect Resource) is a public, computer-readable side effect resource that contains information on marketed medicines and their recorded adverse drug reactions [15]. The information is extracted from public documents and package inserts, in particular, from the US FDA Structured Product Labels (SPLs). The current version was released on October 17, 2012.

PharmGKB DDI-ADE Dataset is a database of DDI side effects based on FDA AERS reporting data [16], in which the confounding factors for prediction of the side effects are corrected through leveraging covariates in observational clinical data [17].

\section{Semantic Web technologies}

The World Wide Web consortium (W3C) is the main standards body for the World Wide Web [18]. The goal of the W3C is to develop interoperable technologies and tools as well as specifications and guidelines to lead the web to its full potential. The resource description framework (RDF), web ontology language (OWL), and SPARQL (a recursive acronym for SPARQL Protocol and RDF Query Language) specifications have all achieved the level of $\mathrm{W} 3 \mathrm{C}$ recommendations, and are becoming generally accepted and widely used. RDF is a model of directed, labeled graphs that use a set of triples. Each triple is modeled in the form of subject, predicate and object. SPARQL is a standard query language for RDF graphs. OWL is a standard ontology language used for ontology modeling.

\section{Methods}

We utilized a normalized AERS dataset known as AERS-DM that was produced in a previous study [11]. The dataset contains 4,639,613 unique putative Drug-ADE pairs in

Table 2 Grade definitions in the CTCAE grading system

\begin{tabular}{ll}
\hline Grade & Definition \\
\hline Grade 1 & $\begin{array}{l}\text { Mild; asymptomatic or mild symptoms; clinical or diagnostic observations only; intervention } \\
\text { not indicated. }\end{array}$ \\
Grade 2 & $\begin{array}{l}\text { Moderate; minimal, local or noninvasive intervention indicated; limiting age-appropriate } \\
\text { instrumental ADL* }\end{array}$ \\
Grade 3 & $\begin{array}{l}\text { Severe or medically significant but not immediately life-threatening; hospitalization or } \\
\text { prolongation of hospitalization indicated; disabling; limiting self care ADL }\end{array}$ \\
Grade 4 & Life-threatening consequences; urgent intervention indicated. \\
Grade 5 & Death related to AE.
\end{tabular}

Note: Activities of Daily Living (ADL); *Instrumental ADL refer to preparing meals, shopping for groceries or clothes, using the telephone, managing money, etc.; **Self care ADL refer to bathing, dressing and undressing, feeding self, using the toilet, taking medications, and not bedridden. 
which the drugs are represented by RxNorm codes and the putative ADEs are represented by MedDRA codes. The AERS-DM dataset is organized in two database files in the Tab Separated Values (TSV) format and accessible at: http://informatics.mayo.edu/ adepedia/index.php/Download.

Figure 1 shows the system architecture of our approach. We first extracted a subset of putative DDI-ADE pairs (in which only two drugs are listed on a report) with their associated outcome codes from original AERS-DM dataset.

Second, we developed a filtering pipeline that comprises three datasets. The first dataset is a subset of original AERS-DM in which only one drug is listed on a report. This dataset was used to build a knowledge base of severe ADEs in a previous study. The second dataset is the SIDER 2 dataset. Table 3 shows a list of drug-ADE pair examples from the dataset, in which drug names are coded in STICH ID (http://stitch. embl.de) and ADE names are coded in MedDRA. We excluded the putative DDI-ADE pairs based on the Drug-ADE pairs of the two datasets. The filtering would ensure that the reported ADEs could not be explained by a single drug effect. The third dataset is a PharmGKB dataset that is used as "silver" standard. Table 4 shows a list of DDI-ADE examples from the dataset, in which drug names are coded in STICH ID and ADE names are coded in UMLS Concept Unique Identifiers (CUIs).

Third, we converted all the datasets used in this study into the Semantic Web RDF format and loaded them into an open source RDF store known as 4store [19]. We established a SPARQL endpoint that provides standard query services against the RDF store. And then we developed the extraction and filtering algorithms using Java-based Jena ARQ APIs [20].

Third, to enrich the signals of the DDI-induced ADEs, we used the NLP-processed EMR data of a cohort of $138 \mathrm{k}$ patients with health home care provided by Mayo Clinic

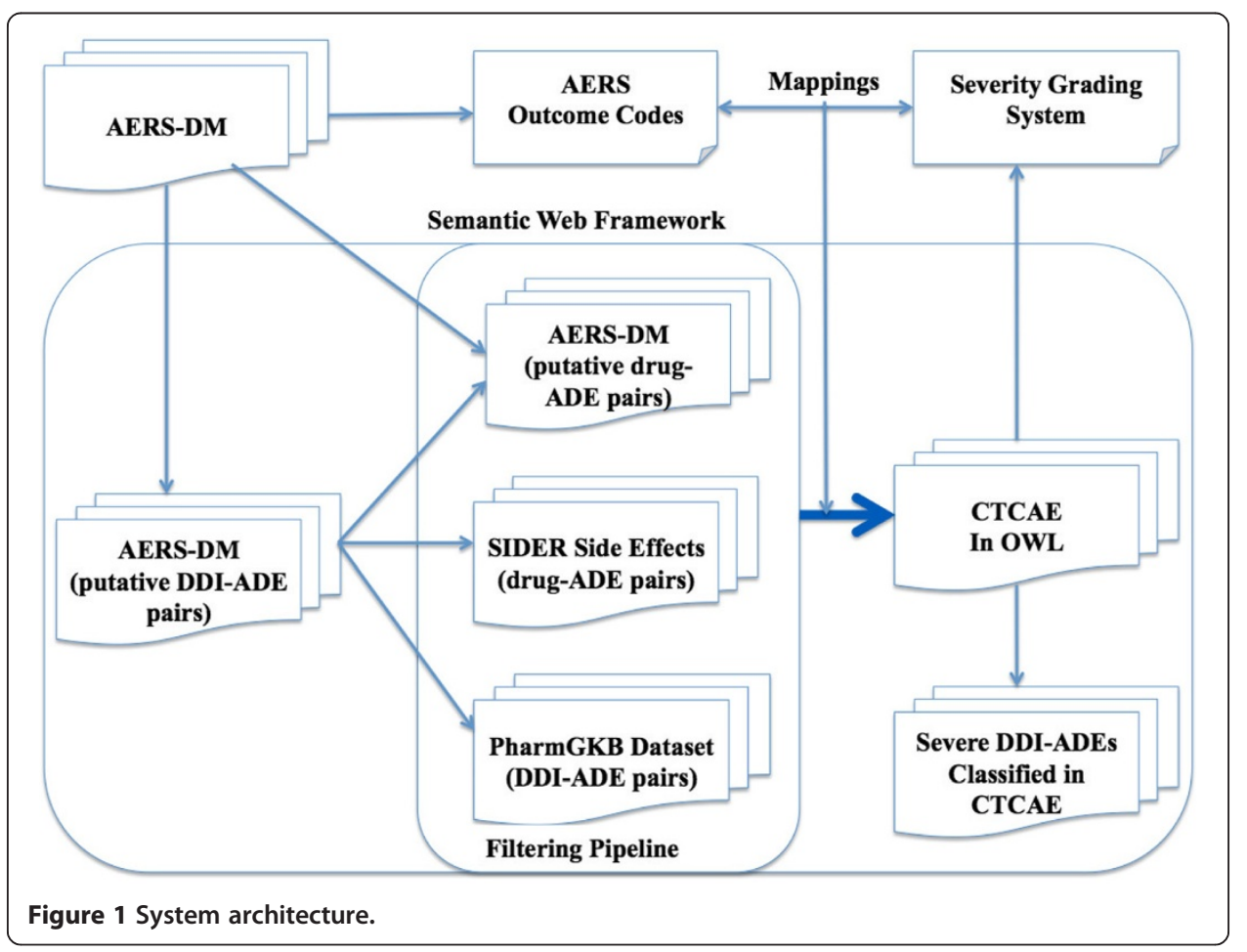


Table 3 A list of Drug-ADE examples from SIDER dataset, in which drug names are coded in STICH ID and ADE names are coded in MedDRA

\begin{tabular}{|c|c|c|c|c|c|c|c|}
\hline stitch_id1 & stitch_id2 & UMLS_con cept_id & Drug_name & side_effect_name & MedDRA_conscept_type & UMLS_concept_id & MEDDRA_side_effect_name \\
\hline-100003914 & -39468 & C0038454 & Levobunolol & cerebrovascular accident & LLT & C0038454 & Cerebrovascular accident \\
\hline-100003914 & -39468 & C0038454 & Levobunolol & cerebrovascular accident & PT & C0038454 & Cerebrovascular accident \\
\hline-100003914 & -39468 & C0015230 & Levobunolol & rash & LLT & C0038454 & Rash \\
\hline-100003914 & -39468 & C0015230 & Levobunolol & rash & PT & C0015230 & Rash \\
\hline-100003914 & -39468 & C0015230 & Levobunolol & rash & PT & C0015230 & Dermatitis \\
\hline-100003914 & -39468 & C0033377 & Levobunolol & ptosis & LLT & C0011603 & Ptosis \\
\hline-100003914 & -39468 & C0033377 & Levobunolol & ptosis & PT & C0033377 & Eyelid ptosis \\
\hline-100003914 & -39468 & C0033377 & Levobunolol & ptosis & PT & C0005745 & Uterovaginal prolapse \\
\hline-100003914 & -39468 & C0030554 & Levobunolol & paresthesia & LLT & C0156353 & Paraesthesia \\
\hline-100003914 & -39468 & C0030554 & Levobunolol & paresthesia & PT & C0030554 & Paraesthesia \\
\hline-100003914 & -39468 & C0006266 & Levobunolol & bronchospas & LLT & C0006266 & Bronhospasm \\
\hline-100003914 & -39468 & C0006266 & Levobunolol & bronchospas & PT & C0006266 & Bronhospasm \\
\hline-100003914 & -39468 & C1145670 & Levobunolol & respiratory failure & LLT & C1145670 & Respiratory failure \\
\hline-100003914 & -39468 & C1145670 & Levobunolol & respiratory failure & PT & C1145670 & Respiratory failure \\
\hline-100003914 & -39468 & C0027424 & Levobunolol & nasal congestion & LLT & C0027424 & Nasal congestion \\
\hline-100003914 & -39468 & C0027424 & Levobunolol & nasal congestion & PT & C0027424 & Nasal congestion \\
\hline-100003914 & -39468 & C0023380 & Levobunolol & lethargy & LLT & C0023380 & Lethargy \\
\hline-100003914 & -39468 & C0023380 & Levobunolol & lethargy & PT & C0023380 & Lethargy \\
\hline-100003914 & -39468 & C0947912 & Levobunolol & myasthenia & LLT & C0947912 & Mysathenia \\
\hline-100003914 & -39468 & C0947912 & Levobunolol & myasthenia & PT & C0151786 & Muscular weakness \\
\hline
\end{tabular}




\begin{tabular}{|c|c|c|c|c|c|}
\hline stitch_id1 & stitch_id2 & drug1 & drug2 & event_umls_id & event_name \\
\hline CID000000085 & CID000000206 & carnitine & galatose & C0004623 & Bacterial infection \\
\hline CID000000085 & CID000000206 & carnitine & galatose & C0015967 & body temperature increased \\
\hline CID000000085 & CID000000206 & carnitine & galatose & C0018932 & haematochezia \\
\hline CID000000085 & CID000000206 & carnitine & galatose & C0020433 & Bilirubinaemia \\
\hline CID000000085 & CID000000206 & carnitine & galatose & C0022346 & icterus \\
\hline CID000000085 & CID000000206 & carnitine & galatose & C0026946 & fungal disease \\
\hline CID000000085 & CID000000206 & carnitine & galatose & C0030305 & panreatitis \\
\hline CID000000085 & CID000000206 & carnitine & galatose & C0040034 & thrombpcytopenia \\
\hline CID000000085 & CID000000206 & carnitine & galatose & C0085605 & Hepatic failure \\
\hline CID000000085 & CID000000206 & carnitine & galatose & C0151766 & Abnormal LFTs \\
\hline CID000000085 & CID000000206 & carnitine & galatose & C0243026 & sepsis \\
\hline CID000000085 & CID000000271 & carnitine & galatose & C0002792 & anaphylactic reaction \\
\hline CID000000085 & CID000000271 & carnitine & galatose & C0002871 & anaemia \\
\hline CID000000085 & CID000000271 & carnitine & galatose & C0002962 & angina \\
\hline CID000000085 & CID000000271 & carnitine & galatose & C0004238 & AFIB \\
\hline CID000000085 & CID000000271 & carnitine & galatose & C0010054 & arteriosclerotic disease \\
\hline CID000000085 & CID000000271 & carnitine & galatose & C0010200 & Cough \\
\hline CID000000085 & CID000000271 & carnitine & galatose & C0012833 & dizziness \\
\hline CID000000085 & CID000000271 & carnitine & galatose & C0013404 & Difficulty breathing \\
\hline CID000000085 & CID000000271 & carnitine & galatose & C0015802 & femur fracture \\
\hline
\end{tabular}

Rochester where medications and problems have been extracted and normalized to RxNorm codes and the UMLS concepts from the current medication and problem list sections of clinical notes using MedXN and MedTagger (http:// www.ohnlp.org/). For each DDI-induced ADE triples (D1, D2, P), we obtained the number of patients who are administrated with any of the two drugs or both (i.e., $\mathrm{N}(\mathrm{D} 1), \mathrm{N}(\mathrm{D} 2)$, and $\mathrm{N}(\mathrm{D} 1, \mathrm{D} 2)$ ) and the number of patients with putative ADEs (i.e., $\mathrm{N}(\mathrm{D} 1, \mathrm{P}), \mathrm{N}(\mathrm{D} 2, \mathrm{P})$, and $\mathrm{N}(\mathrm{D} 1, \mathrm{D} 2, \mathrm{P})$ after taking the drugs. An occurrence of problem $\mathrm{P}$ is considered as putative ADE if it happens within 36 days of drug administration [17] and there is no occurrence of $P$ in the EMR before the drug administration. We then developed the following metric to measure the signal enrichment of DDI-induced ADE:

$$
\operatorname{Score}(D 1, D 2, P)=\log _{2}\left(\frac{N(D 1, D 2, P)}{N(D 1, D 2)} / \max \left(\frac{N(D 1, P)}{N(D 1)}, \frac{N(D 2, P)}{N(D 2)}\right) .\right.
$$

Finally, we developed the mappings between AERS outcome codes and CTCAE grades and classified the filtered DDI-ADEs into the CTCAE. We asserted that DE in AERS corresponds to Grade 5 in CTCAE; LT corresponds to Grade 4; the rest of outcome codes (HO, DS, CA, RI and OT) correspond to Grade 3. In this study, we utilized the CTCAE version 4.0 [14] rendered in OWL format. Figure 2 shows a screenshot of a Protégé4 environment displaying the categories and severity grades in CTCAE classification. 


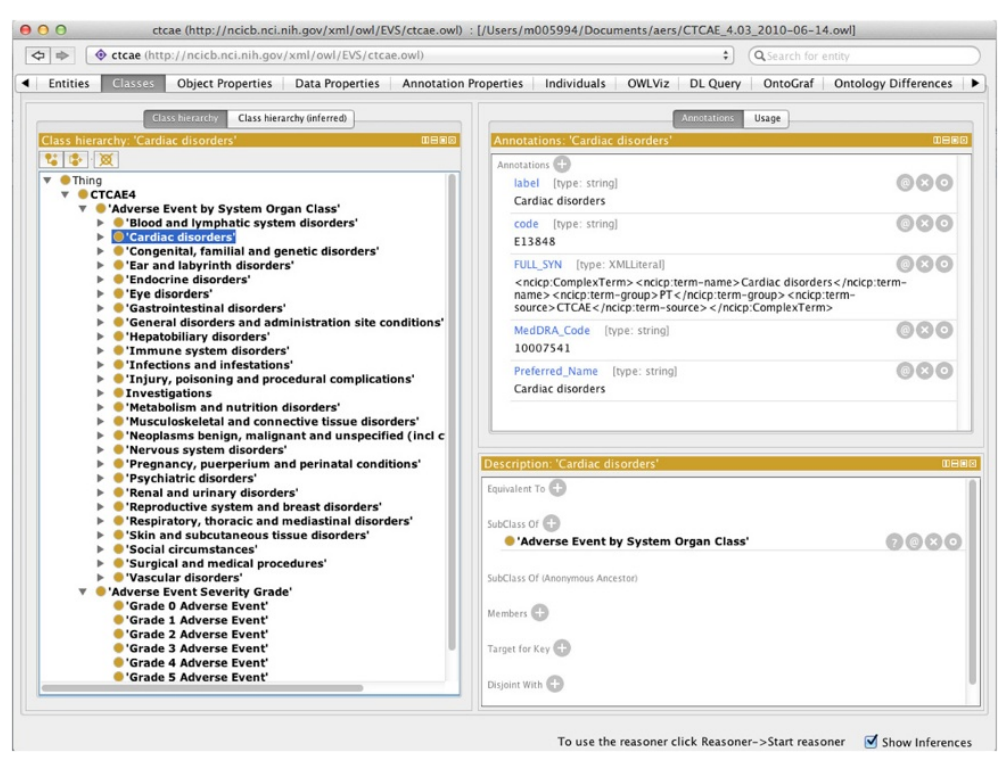

Figure 2 The categories and severity grades of CTCAE classification in a Protégé 4 environment.

\section{Results}

We were able to extract a set of putative DDI-ADE pairs and their associated outcome codes for the three target drugs: Warfarin, Clopidogrel and Simvastatin from normalized AERS-DM dataset. We then filtered the putative DDI-ADE pairs using the filtering pipeline based on three datasets. Table 5 shows the number of filtered DDI-ADE pairs for each target drug. In total, 601 pairs were filtered. Of them, 61 pairs are classified in Grade 5, 56 pairs in Grade 4 and 484 pairs in Grade 3. Table 6 shows a list of filtered DDI-ADE pair examples for the drug "Simvastatin", in which, drugs are coded in RxNorm RxCUIs and ADEs are coded in MedDRA codes.

For the signal enrichment using the EMR data, we found that, there are 89 drug pairs prescribed concomitantly in $9.5 \mathrm{k}$ patients, accounting for $6.9 \%$ of all patients in the EMR dataset we used. Out of 601 putative DDI-ADE pairs, the signals of 59 (D1, D2, P) pairs were identified. Table 7 shows the detailed statistics of those pairs occurred in no less than five patients.

For integrating the filtered DDI-ADE pairs with the CTCAE, we produced an OWL rendering for each pair, asserting the filtered DDI-ADEs under AE terms in CTCAE (see Figure 3 for an example).

Table 5 The number of filtered DDI-ADE pairs for three drugs

\begin{tabular}{llll}
\hline Drug & Number of DDI-ADE Pairs & & Grade 3 \\
\cline { 2 - 4 } & Grade 5 & Grade 4 & 157 \\
\hline Warfarin & 32 & 11 & 166 \\
Clopidogrel & 17 & 29 & 161 \\
Simvastatin & 12 & 16 & 484 \\
Total & 61 & 56 & \\
\hline
\end{tabular}


Table 6 A list of filtered DDI-ADE pairs for the drug "Simvastatin" classified by CTCAE grades

\begin{tabular}{|c|c|c|c|c|c|c|c|}
\hline CTCAE grade & AERS outome code & Drug code by RxCUI & Drug name & Drug code by RxCUI & Drug name & ADE code by MedDRA & ADE name \\
\hline Grede 5 & $\mathrm{DE}$ & 36567 & Simvastatin & 1191 & Aspirin & 10002906 & Aortic stenosis \\
\hline Grede 5 & $\mathrm{DE}$ & 253198 & Risiglitazone maleate & 36567 & Simvastatin & 10006580 & Bundle branch block left \\
\hline Grede 5 & $\mathrm{DE}$ & 36567 & Simvastatin & 203160 & Losartan Potassium & 10007515 & Cardiac arrest \\
\hline Grede 5 & $\mathrm{DE}$ & 36567 & Simvastatin & 1191 & Aspirin & 10010071 & Coma \\
\hline Grede 5 & $\mathrm{DE}$ & 253198 & Risiglitazone maleate & 36567 & Simvastatin & 10012689 & Diabetic retinoathy \\
\hline Grede 4 & LT & 36567 & Simvastatin & 203029 & Tegretol & 10002948 & Aphasia \\
\hline Grede 4 & LT & 36567 & Simvastatin & 203029 & Tegretol & 10003119 & Arrhythmia \\
\hline Grede 4 & LT & 253198 & Amiodarone hydrochloride & 316675 & Simvastatin $80 \mathrm{MG}$ & 10006002 & Bone pain \\
\hline Grede 4 & LT & 36567 & Simvastatin & 225807 & exelon & 10007515 & Cardiac arrest \\
\hline Grede 4 & LT & 36567 & Simvastatin & 203029 & Tegretol & 10012455 & Dematitis exfoliative \\
\hline Grede 3 & DS & 36567 & Simvastatin & 1191 & Aspirin & 10012455 & Dematitis exfoliative \\
\hline Grede 3 & DS & 36567 & Simvastatin & 190465 & Viagra & 10018429 & Glucose tolerance impaired \\
\hline Grede 3 & DS & 36567 & Simvastatin & 83367 & Atorvastatin & 10020765 & Hypersomia \\
\hline Grede 3 & DS & 36567 & Simvastatin & 35296 & Ramipril & 10050295 & Intervertebral disc protrusion \\
\hline Grede 3 & DS & 253198 & Gemfibrozil 600 MG & 316675 & Simvastatin $80 \mathrm{MG}$ & 10000486 & Acidosis \\
\hline
\end{tabular}


Table 7 A list of putative DDI-ADE pairs signaled in the EMR data

\begin{tabular}{|c|c|c|c|c|c|c|c|c|c|c|}
\hline D1 (RxCUI) & D2(RxCUI) & P (MedDRA) & ADE Name & $N(D 1)$ & $N(D 2)$ & $\mathrm{N}(\mathrm{D} 1, \mathrm{D} 2)$ & $N(D 1, P)$ & $\mathrm{N}(\mathrm{D} 2, \mathrm{P}$ & $\mathrm{N}(\mathrm{D} 1, \mathrm{D} 2, \mathrm{P})$ & Score(DI,D2,P) \\
\hline Aspirin (1191) & Simvastatin (36567) & 10002906 & Aortic stenosis & 38149 & 7494 & 2926 & 104 & 34 & 15 & 4.991 \\
\hline Zocor (196503) & Simvastatin (36567) & 10038428 & Renal disorder & 10894 & 7494 & 1472 & 40 & 56 & 7 & 4.550 \\
\hline Simvastatin (36567) & atorvastatin (83367) & 10028417 & Myasthenia gravis & 7494 & 2841 & 828 & 42 & 10 & 5 & 4.409 \\
\hline Warfarin (11289) & Digoxin (3407) & 10013887 & Dysathria & 6330 & 1927 & 641 & 43 & 7 & 6 & 4.36 \\
\hline Aspirin (1191) & Simvastatin (36567) & 10015090 & Epistaxis & 38149 & 7494 & 2926 & 126 & 28 & 9 & 4.257 \\
\hline gabapentin (25480) & Simvastatin (36567) & 10019245 & Hearing impsored & 4683 & 7494 & 280 & 35 & 70 & 5 & 3.935 \\
\hline Plavix (174742) & Simvastatin (36567) & 10017955 & Gastrointestinal heamorrhage & 4769 & 7494 & 642 & 54 & 42 & 9 & 3.88 \\
\hline Aspirin (1191) & clopidogrel (32968) & 10037423 & Pulmunary oedema & 38149 & 1436 & 1291 & 142 & 8 & 8 & 3.338 \\
\hline Aspirin (1191) & clopidogrel (32968) & 10005191 & Blister Dyspnoea exertion & 38149 & 1436 & 1291 & 135 & 9 & 7 & 3.048 \\
\hline Amlodipine (17767) & Simvastatin (36567) & 10013971 & Dyspnoea exertional & 2786 & 7494 & 561 & 62 & 89 & 11 & 2995 \\
\hline Aspirin (1191) & Simvastatin (36567) & 10047924 & Wheezing & 38149 & 7494 & 2926 & 354 & 73 & 27 & 2.969 \\
\hline Lantus (261551) & Simvastatin (36567) & 10012680 & Diabetic neuropathy & 1883 & 7494 & 329 & 39 & 20 & 5 & 2.63 \\
\hline Aspirin (1191) & clopidogrel (32968) & 10038428 & Renal disorder & 38149 & 1436 & 1291 & 175 & 9 & 6 & 2.452 \\
\hline Lantus (261551) & clopidogrel (32968) & 10040882 & Skin lesion & 38149 & 1436 & 1291 & 269 & 21 & 16 & 2.024 \\
\hline Aspirin (1191) & clopidogrel (32968) & 10046555 & Urinary retention & 38149 & 1436 & 1291 & 292 & 16 & 11 & 1.757 \\
\hline Aspirin (1191) & clopidogrel (32968) & 10061623 & Adverse drug reaction & 38149 & 1436 & 1291 & 368 & 20 & 15 & 1.549 \\
\hline Simvastatin (36567) & Norvasc (58927) & 10017076 & fracture & 7494 & 3416 & 318 & 139 & 59 & 6 & 1.219 \\
\hline
\end{tabular}

D1 - drug1, D2 - drug 2, P - problem, N - number, and Score - enrichment score. 


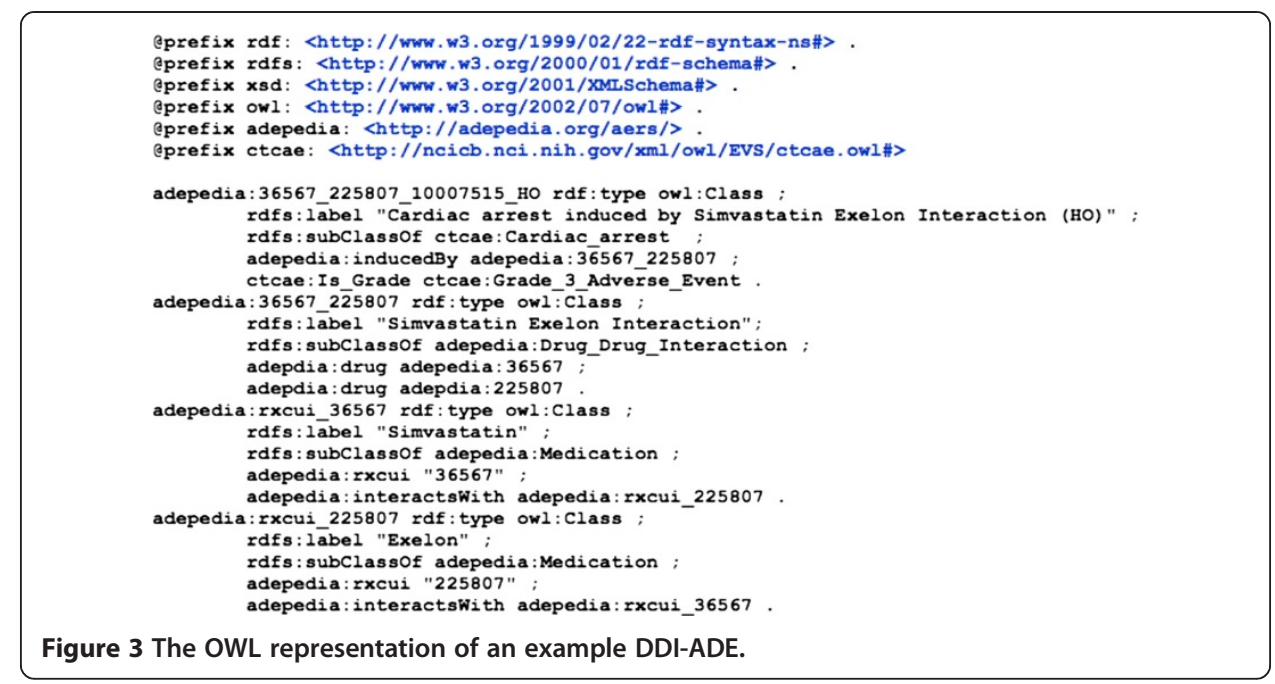

\section{Discussion}

In a previous study, we used a similar Semantic Web-based approach to build a knowledge base of severe ADEs using the FDA AERS reporting data [8]. In this study, we focused on mining the DDI-induced ADEs and their severity information, and configured the filtering pipeline differently using a collection of ADE datasets. The standardization of $\mathrm{ADE}$ datasets is essential for enabling interoperability and comparability among heterogeneous data sources. We used a normalized AERS dataset, in which the drug names are normalized using standard drug ontologies RxNorm and NDF-RT and the ADEs are normalized using MedDRA, whereas the datasets from SIDER and PharmGKB used STITCH compound IDs to code drug names and used UMLS CUIs to code ADEs. Apparently, the solid mappings between RxNorm codes and STITCH IDs would be required in future, which will be part of our research efforts in constructing a standardized drug and pharmacological class network [21].

We also tested the signals of putative DDI-ADE pairs filtered by the pipeline using a large EMR data. We were able to detect some strong signals indicated by the enrichment score as illustrated in Table 7. This would potentially provide a very useful tool for the knowledge-driven detection of the DDI-induced ADEs from the EMR, though a rigorous patient chart review with a panel of clinicians would be needed in future to verify the signals to establish the causality of the drug-drug interaction.

For measuring the severity of ADEs, we used the CTCAE severity grading system. We found that the AERS outcome codes used to record serious patient outcomes in the AERS reporting data correspond well to the CTCAE Grades 3 to 5. Semantic Web OWL rendering of the DDI-ADE dataset provides seamless integration with the CTCAE itself, enabling a standard infrastructure for automatic classification of ADEs based on the severity conditions specified in the CTCAE.

There are several limitations in this study. First, we used the logic that a putative DDI-ADE combination is extracted if there exists an AERS report involving two drugs and the ADE. We understand that the AERS reports themselves do not make it easy to report concomitant drugs and these are known to be under-reported. This means the putative DDI-ADE pairs extracted in this study only reflect a portion of all DDI interactions and should not be considered as a comprehensive list. Second, the PharmGKB 
"silver standard" itself contains signals that have not been validated for causality. This is part of the reasons why we introduce the EMR-based signal enrichment metric in this study. Third, some signals identified from EMR data may not be valid and further rigorous validation approach will be needed in future to filter them out.

\section{Conclusions}

In summary, we developed a Semantic Web-based approach to mine severe DDIinduced ADEs. The dataset produced in this study will be publicly available from our ADEpedia website (http://adepedia.org). The approach developed could be generalized to detect the signals from EMR for putative severe ADEs induced by DDIs in other drug domains and would be useful for supporting translational and pharmacovigilance study of severe ADEs.

\section{Consent}

Informed consent of the use of EMRs for general research was provided by each subject with charts being included in the study. The study was approved by the Institutional Review Committee of the Mayo Clinic as Exempt (Mayo IRB Number: 12-009059).

\section{Competing interests}

The authors declare that they have no competing interests.

\section{Authors' contributions}

All co-authors are justifiably credited with authorship, according to the authorship criteria. Final approval is given by each co-author. In details: GJ - conception, design, development, analysis of data, interpretation of results, and drafting of the manuscript; $\mathrm{HL}$ - analysis of EMR data, interpretation of results and critical revision of the manuscript; HRS - conception and critical revision of the manuscript; CGC - institutional support and critical revision of the manuscript. All authors read and approved the final manuscript.

\section{Acknowledgements \\ The study is supported in part by the SHARP Area 4: Secondary Use of EHR Data (90TR000201).}

Received: 1 August 2014 Accepted: 26 February 2015

Published online: 25 March 2015

References

1. Becker ML, Kallewaard M, Caspers PW, Visser LE, Leufkens HG, Stricker BH. Hospitalisations and emergency department visits due to drug-drug interactions: a literature review. Pharmacoepidemiol Drug Saf. 2007;16(6):64151.

2. Daly AK. Pharmacogenomics of adverse drug reactions. Genome med. 2013;5(1):5

3. Wang L, McLeod HL, Weinshilboum RM. Genomics and drug response. N Engl J Med. 2011;364(12):1144-53.

4. Phillips KA, Veenstra DL, Oren E, Lee JK, Sadee W. Potential role of pharmacogenomics in reducing adverse drug reactions: a systematic review. JAMA. 2001;286(18):2270-9.

5. Percha B, Altman RB. Informatics confronts drug-drug interactions. Trends Pharmacol Sci. 2013;34(3):178-84.

6. Samwald M, Freimuth R, Luciano JS, Lin S, Powers RL, Marshall MS, et al. An RDF/OWL Knowledge Base for Query Answering and Decision Support in Clinical Pharmacogenetics. Stud Health Technol Inform. 2013;192:539-42.

7. Jiang G, Solbrig HR, Chute CG, ADEpedia. A scalable and standardized knowledge base of adverse drug events using Semantic Web technology. AMIA Annu Symp Proc. 2011;2011:607-16.

8. Jiang G, Wang L, Liu H, Solbrig HR, Chute CG. Building a knowledge base of severe adverse drug events based on AERS reporting data using Semantic Web technologies. Stud Health Technol Inform. 2013;192:496-500.

9. Jiang G, Liu HF, Solbrig HR, Chute CG. ADEpedia 2.0: Integration of Normalized Adverse Drug Events (ADEs) Knowledge from the UMLS. AMIA Jt Summits Transl Sci Proc. 2013 Mar 18;2013:100-4.

10. The FDA AERS. [cited June 4, 2013]; Available from: http://www.fda.gov/Drugs/ GuidanceComplianceRegulatoryInformation/Surveillance/AdverseDrugEffects/default.htm.

11. Wang L, Jiang G, Li D, Liu H. Standardizing adverse drug event reporting data. J Biomed Semantics. 2014 Aug 12;5:36. doi: 10.1186/2041-1480-5-36.

12. Nelson SJ, Zeng K, Kilbourne J, Powell T, Moore R, Normalized names for clinical drugs. RxNorm at 6 years. JAMIA. 2011;18(4):441-8

13. The MedDRA. [cited November 16, 2012]; Available from: http://www.meddramsso.com/

14. The CTCAE v4.0. [cited June 1, 2013]; Available from: http://evs.nci.nih.gov/ftp1/CTCAE/About.html.

15. Kuhn M, Campillos M, Letunic I, Jensen LJ, Bork P. A side effect resource to capture phenotypic effects of drugs. Mol Syst Biol. 2010;6:343.

16. PharmGKB Dataset. [cited April 8, 2013]; Available from: http://www.pharmgkb.org/downloads.jsp. 
17. Tatonetti NP, Fernald GH, Altman RB. A novel signal detection algorithm for identifying hidden drug-drug interactions in adverse event reports. JAMIA. 2012;19(1):79-85.

18. The World Wide Web Consortium (W3C). [cited May 25, 2013]; Available from: http:/www.w3.org/.

19. Duke JD, Li X, Grannis SJ. Data visualization speeds review of potential adverse drug events in patients on multiple medications. J Biomed Inform. 2010;43(2):326-31.

20. Ross CJ, Visscher H, Sistonen J, Brunham LR, Pussegoda K, Loo TT, et al. The Canadian Pharmacogenomics Network for Drug Safety: a model for safety pharmacology. Thyroid. 2010;20(7):681-7.

21. Zhu Q, Jiang G, Wang L, Chute CG. Standardized Drug and Pharmacological Class Network Construction. ICBO 2013 - Vaccine and Drug Ontology Studies (VDOS-2013) Workshop. Montreal, Qc. Canada; 2013.

\section{Submit your next manuscript to BioMed Central} and take full advantage of:

- Convenient online submission

- Thorough peer review

- No space constraints or color figure charges

- Immediate publication on acceptance

- Inclusion in PubMed, CAS, Scopus and Google Scholar

- Research which is freely available for redistribution 